\title{
Cross-sections and branching ratios for charged Higgs searches
}

\section{André Sopczak*}

Lancaster / Uppsala University

E-mail: andre.sopczak@cern.ch

For the preparation of the experimental search for charged Higgs bosons at the LHC detailed studies have been made to determine the expected charged Higgs boson production cross-sections and decay branching ratios at $\sqrt{s}=14 \mathrm{TeV}$. In the mass regime below the $t$-quark mass the expected production cross-sections are discussed using PYTHIA and FeynHiggs program packages based on the decay $t \rightarrow H^{+} b$. For higher masses Next-to-Leading-Order (NLO) calculations have been used, and particular attention has been given to the intermediate-mass region. The decay branching ratios have been studied with the program packages FeynHiggs and HDecay. Higher-order corrections $\left(\Delta_{b}\right.$ corrections) in the MSSM are consistently taken into account. Two benchmark scenarios are considered, one of them the 'mhmax'.

Prospects for Charged Higgs Discovery at Colliders

16-19 September 2008

Uppsala, Sweden

\footnotetext{
*Speaker.
} 


\section{Introduction}

Charged Higgs bosons are naturally predicted in non-minimal Higgs scenarios, for instance in Two Higgs Doublet Models (THDMs), and specifically in the Minimal Supersymmetric Standard Model (MSSM) [1].

At present, a lower bound on the charged Higgs boson mass of about $80 \mathrm{GeV}$ exists from LEP [2,3], remarkably close to the previously simulated sensitivity [4]. With initial Tevatron data, upper limits were placed on $\mathrm{BR}\left(t \rightarrow H^{+} b\right)$ for different charged Higgs decay scenarios [5, 6]. Starting from 2009/2010, the LHC at CERN will enable the discovery or the ruling out of the existence of such a particle over a large portion of both the THDM and MSSM parameter space (masses up to around $400 \mathrm{GeV}$ ). The exact value of the reach depends on the value of $\tan \beta$ (reviews [7-9] and a recent study [10]). The expected sensitivities for the LHC have been reported at this workshop $[11,12]$.

This comparative study focuses on the latest developments in the production cross-section and branching ratio determination, and is structured as follows. First the parameter points in the MSSM are defined in section 2. Then in section 3 the determination of the branching ratio $\operatorname{BR}\left(t \rightarrow H^{+} b\right)$ is addressed and results from PYTHIA and FeynHiggs calculations are discussed. This branching ratio is used to determine the $\mathrm{H}^{+}$production cross-section in the low-mass region (section 4). Section 5 addresses the intermediate-mass region around $m_{H^{+}}=170 \mathrm{GeV}$. In the high-mass region the process $g b \rightarrow H^{+}$is dominant and the calculations are described in section 6 . The $\mathrm{H}^{+}$branching ratios are discussed in section 7. Systematic uncertainties are the focus in section 8. Section 9 describes the data-base structure for storing the cross-section and branching ratio values.

\section{MSSM scenarios}

Two scenarios in the MSSM are considered. They are described by the following parameters. Scenario A: $m_{t}=175 \mathrm{GeV}, M_{\mathrm{SUSY}}=500 \mathrm{GeV}, A_{t}=1000 \mathrm{GeV}, \mu=200 \mathrm{GeV}, M_{2}=1000 \mathrm{GeV}$, $M_{3}=1000 \mathrm{GeV}$. Scenario B ("mhmax") [13]: $m_{t}=175 \mathrm{GeV}, M_{\mathrm{SUSY}}=1000 \mathrm{GeV}, X_{t}=2000 \mathrm{GeV}$, where $A_{t}=X_{t}+\mu / \tan \beta, \mu=200 \mathrm{GeV}, M_{2}=200 \mathrm{GeV}, M_{3}=800 \mathrm{GeV}$.

The $\Delta_{b}$ corrections are calculated in FeynHiggs v2.6.2 [14] for these two cases in the $H^{+}$ couplings. For $\tan \beta=50$ they are $\Delta_{b}=0.45$ for scenario A, and $\Delta_{b}=0.21$ for scenario B. The $\Delta_{b}$ corrections modify the $b$-quark mass $m_{b}^{\text {corrected }}=m_{b} /\left(1+\Delta_{b}\right)[15]$.

\section{3. $\mathbf{t} \rightarrow \mathbf{H}^{+} \mathbf{b}$ branching ratios}

The $\mathrm{BR}\left(t \rightarrow H^{+} b\right)$ values have been determined with PYTHIA v6.325 [16] and FeynHiggs v2.6.2 [14] ${ }^{1}$. In FeynHiggs the formula from Ref. [17] is implemented, and furthermore it includes the $\Delta_{b}$ corrections depending on the MSSM parameters [15]. The computations have been performed for MSSM scenarios A and B. An example is shown in Fig. 1 for scenarios A as a function of $\tan \beta$. The FeynHiggs calculations include $\Delta_{b}$ corrections, while the PYTHIA calculation does

\footnotetext{
${ }^{1}$ In this study the branching ratios were also produced with FeynHiggs v2.6, however, then discarded as the differences in version 2.6 and 2.6.2 were only attributed to a programming correction ('bug fix') in the latter version.
} 
not. The $\Delta_{b}$ corrections are positive and grow with $\tan \beta$ which explains the observed increase in $\operatorname{BR}\left(t \rightarrow H^{+} b\right)$ difference with increasing $\tan \beta$ (Fig. 1).

The first step in the determination of the production cross-section is the calculation of the branching ratio $\mathrm{BR}\left(t \rightarrow H^{+} b\right)$ in the low mass region 90 to $170 \mathrm{GeV}$. These branching ratios are also shown in Fig. 1 for scenario A, calculated with FeynHiggs.
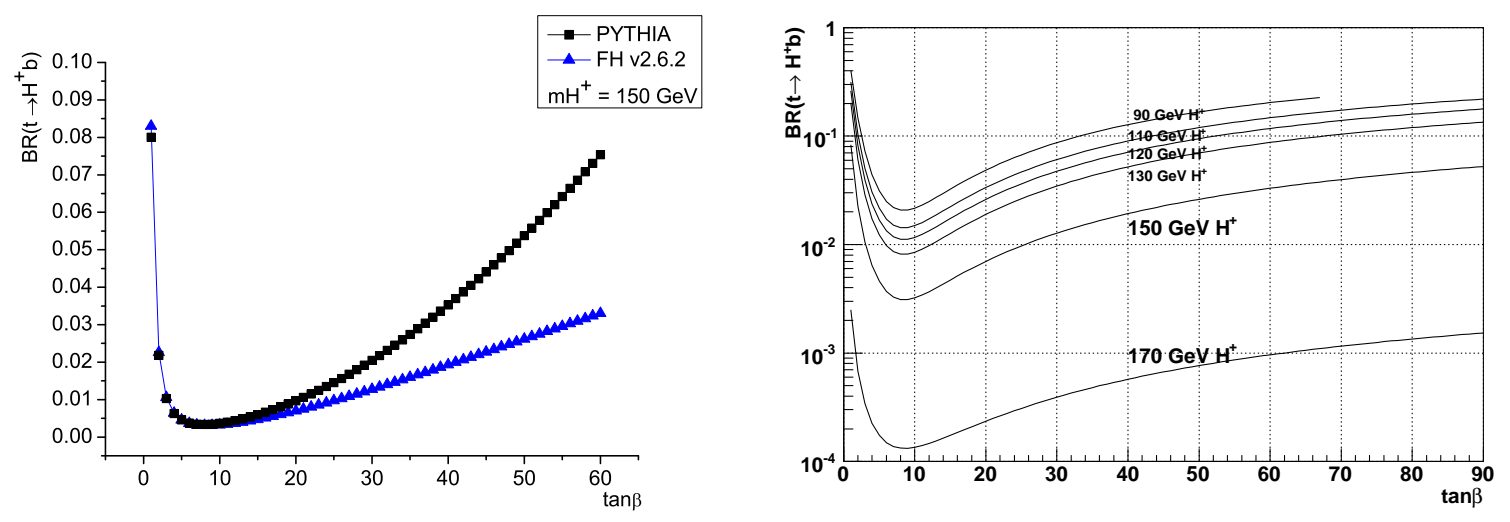

Figure 1: Left: Expected branching ratio $\mathrm{BR}\left(t \rightarrow H^{+} b\right)$ for MSSM scenario A. Right: Expected branching ratio $\mathrm{BR}\left(t \rightarrow H^{+} b\right)$ for MSSM scenario A as described in the text, calculated with FeynHiggs version 2.6.2. For very large $\tan \beta$ values and low charged Higgs masses $(90 \mathrm{GeV})$, the model enters a non-perturbative regime and loop calculations are no longer valid, as indicated by the ending of the $90 \mathrm{GeV}$ curve.

\section{Low-mass cross-section}

The charged Higgs boson production cross-section has been evaluated with different program packages for low-mass and high-mass regions. If the charged Higgs boson mass $m_{H^{+}}$satisfies $m_{H^{+}}<m_{t}-m_{b}$, where $m_{b}$ is the $b$-quark mass, $H^{+}$particles could be produced in the on-shell process $t \rightarrow b H^{+}$, the latter being in turn produced from $g g$ fusion and $q \bar{q}$ annihilation. Contributions of gluon fusion to the production of charged Higgs at hadron colliders were pointed out previously [18]. This approximation has customarily been used in event generators for $m_{H^{+}} \lesssim m_{t}$.

Charged Higgs production is denoted by $q \bar{q}, g g \rightarrow t \bar{t} \rightarrow t b H^{+}$if due to (anti-) top decays and by $q \bar{q}, g g \rightarrow t b H^{+}$if further production diagrams are included [19]. Owing to the large $t$-quark decay width $\left(\Gamma_{t} \simeq 1.5 \mathrm{GeV}\right)$ and to the additional diagrams which do not proceed via direct $t \bar{t}$ production [20-22], charged Higgs bosons could also be produced at and beyond the mass threshold $\left(m_{t}-m_{b}\right)$ in the $t b H^{+}$process. The importance of these effects in the so-called 'threshold' or 'transition' region (intermediate-mass region $m_{H^{+}} \approx m_{t}$ ) was emphasized in various Les Houches proceedings [23, 24] as well as in Refs. [19, 25-27], and the calculations of Refs. [20,21] (based on the appropriate $q \bar{q}, g g \rightarrow t b H^{+}$description) are now implemented in HERWIG [28-31] and PYTHIA [16, 19]. A comparison between the two generators was carried out in Ref. [19]. In addition, in the mass region near the $t$-quark mass, a matching of the calculations for the $q \bar{q}, g g \rightarrow$ $t b H^{+}$and $g b \rightarrow t H^{+}$processes is required already in leading order [32]. In the kinematic region where $t \rightarrow H^{+} b$ is possible, this process could dominate the charged Higgs production.

The cross-sections in the low-mass region for the charged Higgs boson masses 90, 110, 120 , 130 and $150 \mathrm{GeV}$ have been calculated from the higher-order improved $t \bar{t}$ cross-section $\left(\sigma_{t \bar{t}}=\right.$ 
$833 \mathrm{pb}$ [33]) and the $\mathrm{BR}\left(t \rightarrow H^{+} b\right)$ determined from FeynHiggs (version 2.6.2) [14]: $\sigma_{t b H^{+}}=$ $2 \cdot \sigma_{t \bar{t}} \mathrm{BR}\left(t \rightarrow H^{+} b\right)\left[1-\mathrm{BR}\left(t \rightarrow H^{+} b\right)\right]$. Results are shown in Fig. 2 (right plot).

\section{Intermediate-mass cross-section}

Charged Higgs bosons with a mass of $170 \mathrm{GeV}$ would predominantly be produced by the $g b \rightarrow$ $t H^{+}$process. The intermediate-mass region has been studied in NLO [34]. The $t$-quark mass has been fixed to $175 \mathrm{GeV}$. For this scenario with a $5 \mathrm{GeV}$ mass difference between charged Higgs and $t$-quark masses, the additional cross-section from the $t \rightarrow H^{+} b$ process amounts to an increase of about 20 to $30 \%$. We have taken into account this increase in the derivation of the production crosssection by adding both cross-sections. The cross-section increase depends strongly on the mass difference between charged Higgs and $t$-quark masses, and also on the treatment of the running $b$-quark mass. Results are shown in Fig. 2 (right plot, $170 \mathrm{GeV}$ curve).

\section{High-mass cross-section}

At hadron colliders, the main contribution to charged Higgs boson production is through the twin processes $g g \rightarrow t b H^{+}$and $g b \rightarrow t H^{+}$for $m_{H^{+}}>m_{t}$. These are called twin processes since they correspond to two different approximations describing the same basic process. For charged Higgs boson masses above the $t$-quark mass, the $2 \rightarrow 2$ process is dominant, due to the resummation of potentially large logarithms in the $b$-quark parton density [35]. In the high-mass region the Nextto-Leading Order (NLO) production cross-section calculation is applied $[34,35]$. In this case, the parton shower produces an outgoing $b$-quark of relative small transverse momentum. In the region of phase space where the outgoing $b$-quark has large transverse momentum, the parton shower does not give a good description of the process, and the full $2 \rightarrow 3$ description is needed. However, these two descriptions overlap for small transverse momenta of the $b$-quark, necessitating a matching procedure to remove resulting double counting [32]. For charged Higgs boson masses below the $t$-quark mass, the $2 \rightarrow 3$ process dominates since it incorporates on-shell $t$-quark pair production with subsequent decay into a charged Higgs boson. Matchig [36] is a new leading-order event generator based on the work presented in Ref. [32] which matches the two processes by producing negative weight events from an identified double-counting term. In the high-mass region the NLO program package [34] also avoids double counting.

For the charged Higgs boson masses 200, 250, 350, 400, 500 and $600 \mathrm{GeV}$, the $\mathrm{BR}(t \rightarrow$ $\left.\mathrm{H}^{+} b\right)$ is kinematically suppressed and the cross-sections have been determined in NLO from the $g b \rightarrow t H^{+}$process alone [35]. We have explicitly not calculated the dependence of the crosssections on the MSSM parameters with the NLO program package $[34,35]^{2}$. These higher-order corrections can be large, depending on the MSSM scenario. They depend primarily on $\tan \beta$. In order to determine these corrections, first the $\Delta_{b}$ corrections are calculated with the FeynHiggs package [14]. Then a reduction factor $f=1 /\left(1+\Delta_{b}\right)^{2}$ is calculated for the production crosssection. The previously determined NLO cross-sections are multiplied by this reduction factor.

\footnotetext{
${ }^{2}$ Recently, the charged Higgs production process at NLO has been implemented in the program package PROSPINO 2.1 including $\Delta_{b}$ corrections.
} 
The reduction factors for scenario $\mathrm{A}$ are shown in Fig. 2 as a function of $\tan \beta$. The figure shows also the cross-sections after the application of the reduction factors ${ }^{3}$.
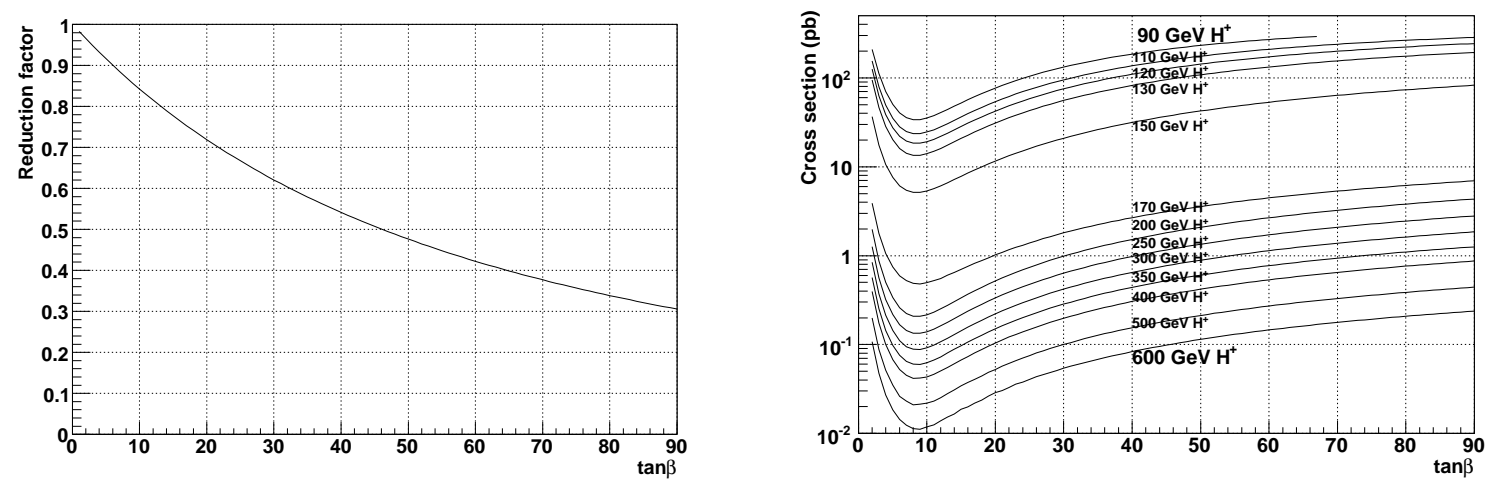

Figure 2: Left: Reduction factors $f$ which have been applied to the NLO charged Higgs boson production cross-section in the MSSM for scenario A. The reduction factors result from $\Delta_{b}$ corrections and have been calculated with FeynHiggs v2.6.2 [14]. Right: Expected charged Higgs boson production cross-section in the MSSM for scenario A. For charged Higgs boson masses of $170 \mathrm{GeV}$ and above the reduction factors $f$ have been applied to the NLO cross-section calculations [35]. This allows a consistent treatment of the $\Delta_{b}$ corrections for the production cross-sections and branching ratios over the whole mass range.

\section{7. $\mathrm{H}^{+}$branching ratios}

This section describes the $\mathrm{H}^{+}$branching ratio studies, in particular focusing on the off-massshell effects and the $\Delta_{b}$ corrections. The $\operatorname{BR}\left(H^{+} \rightarrow \ldots\right)$ values have been determined with the FeynHiggs v2.5, v2.6.2 and HDecay 3.2 program packages. FeynHiggs v2.5 does not include offmass-shell effects while FeynHiggs v2.6.2 does. The difference between FeynHiggs v2.6.2 and HDecay $3.2^{4}$ is that FeynHiggs includes the $\Delta_{b}$ corrections.

While for $m_{H^{+}} \lesssim m_{t}$ charged Higgs bosons decay predominantly into a $\tau$-lepton and a neutrino, or into a $c s$-quark pair; for $m_{H^{+}} \gtrsim m_{t}$ both $H^{+} \rightarrow \tau \nu_{\tau}$ and $H^{+} \rightarrow t b$ are important decay channels. In the experimental search, the latter is much harder to disentangle from background than the former. The associated $t$-quark decays predominantly into a $W$ boson and a $b$-quark.

The branching ratios have been determined with the FeynHiggs [14] and HDecay [38] program packages. The detailed comparison showed very good agreement between FeynHiggs and HDecay calculations for the branching ratios in the low-mass region $\left(m_{H^{+}}<m_{t}\right)$, including the virtual effects which lead to $H^{+} \rightarrow t b$ contribution in this mass region ${ }^{5}$. In the high-mass region, vertex corrections $\left(\Delta_{b}\right.$ terms) which are included in FeynHiggs lead to a significant variation of the branching ratio with $\tan \beta$ while the branching ratios calculated with HDecay are largely independent of $\tan \beta$ in the high-mass region. The branching ratio comparison is given in Table 1 .

Examples of branching ratios are shown in Fig. 3 in the low-mass region for a $130 \mathrm{GeV}$ and in the high-mass region for a $600 \mathrm{GeV}$ charged Higgs boson [14]. The dependence of the branching

\footnotetext{
${ }^{3}$ However, for any sub-dominant decay channel of a heavy charged Higgs boson, such as $H^{+} \rightarrow \tau^{+} v, \Delta_{b}$ corrections cancel to a large extent [37].

${ }^{4}$ Since version $3.3 \Delta_{b}$ corrections are included in HDecay for the charge Higgs sector.

${ }^{5}$ These virtual effects were only included in FeynHiggs version 2.6, release July 2007. In previous versions of FeynHiggs the $t b$ branching fraction due to virtual effects was zero.
} 


\begin{tabular}{|r|rrrrrr|}
\hline Decay mode & $\mathrm{BR}\left(H^{+} \rightarrow \ldots\right) \tau \nu$ & $c s$ & $t b$ & $\tau \nu$ & $c s$ & $t b$ \\
$m_{H^{+}}(\mathrm{GeV})$ & 170 & 170 & 170 & 400 & 400 & 400 \\
\hline $\tan \beta=3, \mathrm{FH}$ & $0.901 / 0.766$ & $0.009 / 0.008$ & $0 / 0.1485$ & $0.004 / 0.004$ & $0.000 / 0.000$ & $0.978 / 0.978$ \\
$3, \mathrm{HD}$ & 0.745 & 0.008 & 0.133 & 0.004 & 0.000 & 0.984 \\
$10, \mathrm{FH}$ & $0.990 / 0.988$ & $0.006 / 0.006$ & $0 / 0.002$ & $0.146 / 0.146$ & $0.001 / 0.001$ & $0.845 / 0.845$ \\
$10, \mathrm{HD}$ & 0.974 & 0.006 & 0.001 & 0.112 & 0 & 0.88 \\
$60, \mathrm{FH}$ & $0.991 / 0.991$ & $0.006 / 0.006$ & $0 / 0.000$ & $0.336 / 0.336$ & $0.002 / 0.001$ & $0.660 / 0.662$ \\
$60, \mathrm{HD}$ & 0.976 & 0.006 & 0 & 0.143 & 0 & 0.854 \\
\hline
\end{tabular}

Table 1: Branching ratios $\mathrm{BR}\left(H^{+} \rightarrow \tau v, c s, t b\right)$ for FeynHiggs (FH), left: v2.5, right v2.6.2, and HDecay (HD) v3.2. Version 2.6.2 includes off-mass-shell effects, while version v2.5 does not. It has been explicitly checked that the difference between FeynHiggs v2.6.2 and HDecay are due to the $\Delta_{b}$ corrections [39].

ratio as a function of the mass is also shown in Fig. 3. When kinematically allowed the decay $H^{+} \rightarrow \chi^{0} \chi^{+}$could become large, as calculated with FeynHiggs v2.6.2. This decay mode is not addressed in the current discovery analyses and this branching fraction is not discussed further.
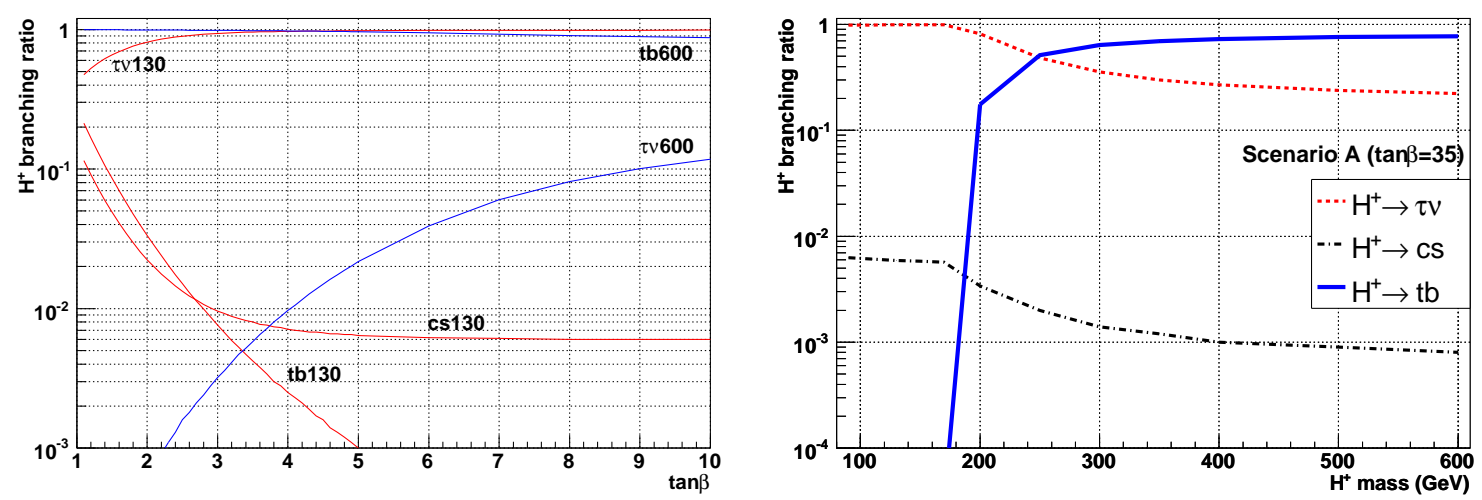

Figure 3: Left: Expected charged Higgs boson branching ratios in the MSSM for scenario A for an example of a light and a heavy charged Higgs boson [14]. Right: Expected charged Higgs boson branching ratios in the MSSM for scenario A as a function of the charged Higgs boson mass [14].

\section{Systematic Uncertainties}

The systematic uncertainties are discussed for the charged Higgs branching ratio $\mathrm{BR}\left(t \rightarrow H^{+} b\right)$ and production cross-section in the low-mass region for $m_{H^{+}}<m_{t}$. The charged Higgs boson branching ratios $\mathrm{BR}\left(H^{+} \rightarrow \tau v, c s, t b\right)$ have been determined with the same program package and similar systematic uncertainties apply. The production cross-sections in the high-mass region have been determined with a NLO program package [35], and the systematic uncertainties are discussed separately.

The values for $\mathrm{BR}\left(t \rightarrow H^{+} b\right)$ and $\mathrm{BR}\left(H^{+} \rightarrow \tau v, c s, t b\right)$ have been calculated with FeynHiggs v2.6.2 which includes vertex corrections in the framework of the MSSM $\left(\Delta_{b}\right.$ terms). Systematic uncertainties from higher-order loop corrections to the $t b H^{+}$vertex and due to the running of the $c$ and $s$-quark masses are expected [39]. Upper limits on the resulting systematic uncertainties are conservatively estimated to be:

$\Delta \Gamma\left(t \rightarrow H^{+} b\right) / \Gamma<10 \%, \Delta \Gamma\left(H^{+} \rightarrow \tau v\right) / \Gamma<5 \%, \Delta \Gamma\left(H^{+} \rightarrow c s, t b\right) / \Gamma<10 \%$. 
Systematic uncertainties on the charged Higgs boson production cross-section in the highmass region can occur primarily from two sources. First from the renormalization scale and factorization scale dependence, and second from the fact that supersymmetry loop corrections are not (yet) included in the calculations [40,41]. The one-loop contributions largely improve the theoretical uncertainty of the leading order (LO) cross-section. The remaining uncertainty can be estimated from the scale dependence. The variation of the $\mathrm{gb} \rightarrow t \mathrm{H}^{+}$production cross-section for $0.1<\mu / \mu_{\text {central }}<10$ was considered, where $\mu_{\text {central }}=\left(m_{t}+m_{H^{+}}\right) / 5$. The resulting systematic uncertainty on the production cross-section is below $20 \%$.

Corrections from supersymmetric particles in the MSSM are not included in the NLO production cross-section calculations $[40,41]$. In this study, these supersymmetry loop corrections $\left(\Delta_{b}\right.$ corrections) have been taken into account independent of the NLO calculations using the FeynHiggs package and thus no additional uncertainty beyond the $20 \%$ is assigned ${ }^{6}$.

\section{Database}

The branching ratio $\mathrm{BR}\left(t \rightarrow H^{+} b\right)$, the charged Higgs boson production cross-section in the low-mass, intermediate-mass and high-mass regions, the $\Delta_{b}$ values and corresponding cross-section reduction factors, and the $\mathrm{H}^{+}$branching ratios have been determined for various charged Higgs boson masses between 90 and $600 \mathrm{GeV}$ and for $\tan \beta$ values between 1 and 90 . The calculated values have been stored in a database in root format for scenarios $\mathrm{A}$ and $\mathrm{B}$.

\section{Conclusions}

Comparative studies for the expected charged Higgs boson production cross-sections and branching rations have been performed for searches in the initial LHC data. The production crosssections in the low-mass region and all charged Higgs branching ratios have been calculated with FeynHiggs v2.6.2. The production cross-sections in the high-mass region have been determined with a dedicated NLO program package and the dependence on MSSM parameters has been added using the FeynHiggs package.

\section{Acknowledgements}

I would like to thank the organizers of the workshop for their hospitality, in particular, Johan Rathsman and Tord Ekelöf. Also I would like to thank Johan Alwall and Oscar Stål for fruitful discussion on the charged Higgs production processes, Tilman Plehn for discussions regarding the NLO cross-section program package, Michal Spira for discussions on the HDecay program package, and Sven Heinemeyer for discussions on the FeynHiggs program package. I also thank the ATLAS csc HG-10 team for discussions, in particular, Thies Ehrich, Martin Flechl, Eilam Gross, Bjarte Mohn and Remi Zaidan.

\footnotetext{
${ }^{6}$ Without the $\Delta_{b}$ corrections, these uncertainties from supersymmetric corrections were in addition. They alter the relation between the bottom mass and the bottom Yukawa coupling. These $\Delta m_{b}$ corrections are the leading supersymmetric one-loop corrections with respect to powers of $\tan \beta$. (The naming convention for $\Delta m_{b}$ has changed to $\Delta_{b}$. They refer to the same corrections.) As pointed out in Ref. [40,41] their effect on the total cross-section in a simple mSUGRA model is estimated to stay below $\pm 5 \%$ for $\tan \beta=30$ and below $\pm 20 \%$ for $\tan \beta=50$. Figure 2 of this study shows that the effect can be larger than $50 \%$ for $\tan \beta>50$ in the MSSM. In addition to the $\Delta m_{b}$ corrections higher-order supersymmetry QCD corrections have been calculated recently [42].
} 


\section{References}

[1] J.F. Gunion, H.E. Haber, G.L. Kane and S. Dawson, The Higgs Hunters' Guide, Addison-Wesley 1990.

[2] ALEPH, DELPHI, L3 and OPAL [The LEP Higgs Working Group], hep-ex/0107031.

[3] A. Sopczak, "Review of current limits from charged Higgs searches at LEP”, Workshop on Prospects for Charged Higgs Discovery at Colliders, Uppsala, September 2006.

[4] A. Sopczak, Int. J. Mod. Phys. A 9 (1994) 1747.

[5] A. Abulencia et al, CDF Collaboration, Phys. Rev. Lett. 96 (2006) 042003.

[6] P. Gutierrez, "Charged Higgs searches with D0", these proceedings.

[7] D.P. Roy, Mod. Phys. Lett. A 19 (2004) 1813.

[8] D.P. Roy, hep-ph/0409201.

[9] D.P. Roy, hep-ph/0510070.

[10] B. Mohn, M. Flechl and J. Alwall, ATL-PHYS-PUB-2007-006 (2007).

[11] M. Flechl, "Charged Higgs prospects with ATLAS", these proceedings.

[12] R. Kinnunen, "Charged Higgs prospects with CMS", these proceedings.

[13] M.S. Carena, S. Heinemeyer, C.E.M. Wagner and G. Weiglein, Eur. Phys. J. C 26 (2003) 601.

[14] FeynHiggs version 2.6.2, S. Heinemeyer et al., and private communications, hep-ph/0611326, hep-ph/0212020, hep-ph/9812472, hep-ph/9812320.

[15] M. Carena, D. Garcia, U. Nierste and C. Wagner, Nucl. Phys. B 577 (2000) 88.

[16] T. Sjöstrand, Comput. Phys. Comm. 82 (1994) 74.

[17] J.G. Körner and M.C. Mauser, hep-ph/0211098.

[18] J.L. Diaz-Cruz and O.A. Sampayo, Phys. Rev. D 50 (1994) 6820.

[19] J. Alwall, C. Biscarat, S. Moretti, J. Rathsman and A. Sopczak, Eur. Phys. J. C 39S1 (2005) 37.

[20] F. Borzumati, J.-L. Kneur and N. Polonsky, Phys. Rev. D 60 (1999) 115011.

[21] D.J. Miller, S. Moretti, D.P. Roy and W.J. Stirling, Phys. Rev. D 61 (2000) 055011.

[22] S. Moretti and D. P. Roy, Phys. Lett. B 470 (1999) 209.

[23] D. Cavalli et al., hep-ph/0203056.

[24] K.A. Assamagan et al. [Higgs Working Group Collaboration], hep-ph/0406152.

[25] M. Guchait and S. Moretti, JHEP 0201 (2002) 1.

[26] S. Moretti, Pramana 60 (2003) 369.

[27] K.A. Assamagan, M. Guchait and S. Moretti, hep-ph/0402057.

[28] G. Marchesini et al., Comput. Phys. Commun. 67 (1992) 465.

[29] G. Corcella et al., JHEP 101 (2001) 10.

[30] G. Corcella et al., hep-ph/0210213.

[31] S. Moretti, K. Odagiri, P. Richardson, M.H. Seymour and B.R. Webber, JHEP 204 (2002) 28.

[32] J. Alwall and J. Rathsman, JHEP 12 (2004) 50.

[33] R. Bonciani, S. Catani, M.L. Mangano and P. Nason, Nucl. Phys. B 529 (1998) 424

[Erratum-ibid. B 803, 234 (2008) 234].

[34] E.L. Berger, T. Han, J. Jiang and T. Plehn, Phys. Rev. D 71 (2005) 115012.

[35] E. Boos and T. Plehn, Phys. Rev. D 69 (2004) 094005, and references therein

[36] J. Alwall, hep-ph/0503124 (2005).

[37] D. Eriksson, F. Mahmoudi and O. Stål, JHEP 0811 (2008) 35.

[38] A. Djouadi, J. Kalinowski and M. Spira, Comput. Phys. Commun. 108 (1998) 56.

[39] S. Heinemeyer, private communications (2007).

[40] T. Plehn, Phys. Rev. D 67 (2003) 014018.

[41] S.-H. Zhu, Phys. Rev. D 67 (2003) 075006.

[42] D. Noth and M. Spira, Phys. Rev. Lett. 101 (2008) 181801. 